Vierteljahrsschrift für Geschichte der Medizin und der Naturwissenschaften Revue trimestrielle d'histoire de la médecine et des sciences naturelles Redaktion/Rédaction: Hans H. Walser, Zürich Heinz Balmer, Zürich
GESNERUS
Jahrgang/Vol.36 1979

Heft / Fasc. 1/2

Verlag/Editions Sauerländer

\title{
Johannes von Muralts «Geburts-Tafel»
}

Zur Geschichte der Berechnung des Geburtstermins

Von Urs Boschung

Die Medizinhistorische Sammlung der Universität Zürich besitzt als Unikum einen Schwangerschaftskalender, den der Stadtarzt Johannes von Muralt im Jahre 1701 dem Ehegericht der Stadt und Landschaft Zürich geschenkt hat ${ }^{1}$.

Johannes von Muralt (1645-1733) entstammte einer 1566 in Zürich eingebürgerten, ursprünglich in Locarno ansässigen Familie. Während seines Medizinstudiums in Basel, Leiden, Paris und Montpellier widmete er sich der Anatomie, aber auch der Chirurgie, die er nach der Rückkehr in seine Vaterstadt Zürich gegen den Widerstand der Meister des Schererhandwerks ausübte. Vom Rat erkämpfte sich Muralt die Erlaubnis, zur Belehrung von Chirurgen und Ärzten anatomische Sektionen auszuführen. Seit 1688 Stadtarzt, nahm er sich auch der Hebammenausbildung an. In seinen Schriften erweist er sich als vielseitiger biologischer Forscher und erfahrener Arzt, Chirurg und Geburtshelfer ${ }^{2}$.

\section{Der Schwangerschaftskalender}

Bei Muralts Schwangerschaftskalender handelt es sich um eine $114 \mathrm{~cm}$ hohe und $38,5 \mathrm{~cm}$ breite Holztafel, die in einen $6,5 \mathrm{~cm}$ breiten, schwarz gestrichenen Holzrahmen mit vergoldeter Zierleiste gefaßt ist (Abb. 1). Die Namen und Wappen der Mitglieder des Ehegerichts nehmen die zwei oberen Drittel, die eigentliche «Geburts-Tafel»den unteren Teil ein (Abb. 2). Diese besteht aus vier konzentrischen Kreisen, auf denen abgetragen sind (von außen nach innen):

1. 12 Monate des Sonnenjahres mit 365 Tagen; je sieben Tage bezeichnet mit a bis $\mathrm{g}$, entsprechend den sieben Wochentagen $(\varnothing 37 \mathrm{~cm})$, 
2. zehn Monate des Mondjahres mit 295 Tagen $(\varnothing 28 \mathrm{~cm})$,

3. zehn Monate der Schwangerschaft mit 300 Tagen $(\varnothing 23 \mathrm{~cm})$,

4. sieben vierzigtägige Abschnitte mit 280 Tagen $(\varnothing 18,5 \mathrm{~cm})$.

Die drei inneren Kreise sind auf drei Scheiben aus Eisenblech gemalt, die sich um eine zentrale Schraube drehen und durch eine Flügelmutter fixieren lassen. Durch dieselbe Schraube werden auch die vier Zeiger aus Messing (Länge je $15 \mathrm{~cm}$ ) gehalten. Drei von ihnen sind fest miteinander verbunden. Sie tragen die Beschriftung «Anfang der Schwangerschaft», «Halbe Jahrs-Zeit» und «Die von der Natur bestimte Geburts-Zeit». Der vierte Zeiger ist frei beweglich («GeburtsZeit»). Die drei ersten Zeiger bilden miteinander konstante Winkel von etwa 180 und 270 Grad. Sie erlauben, auf dem Kalenderkreis Zeitintervalle von 180 Tagen, d.h. einem halben Jahr, und von 278 Tagen $^{3}$, entsprechend der angenommenen Schwangerschaftsdauer, zu bestimmen.

Hebt man die oberste, kleinste Scheibe ab, auf der eine schlafende allegorische Figur mit dem Schriftband «Memento Mori» dargestellt ist, so kommt auf der zweiten Scheibe die Signatur des Malers Johann Melchior Füßli zum Vorschein (Abb. 3). Füßli, geboren in Zürich 1677, gestorben ebenda 1736, stammt aus der weitverzweigten bekannten Künstlerfamilie. Er bildete sich in Zürich und Berlin zum Maler und Kupferstecher aus. In Zürich, wo er sich 1697 niederließ, illustrierte er unter anderem mehrere Reihen von Neujahrsblättern ${ }^{4}$. Viel bedeutender ist Füßlis Arbeit für Johann Jakob Scheuchzer (1672-1733), der ihn seinen «werthesten Freund» nennt. So lieferte er für Scheuchzers «Kupfer-Bibel», die in den 1730 er Jahren in mehreren Sprachen erschien, die Vorlagen zu den 750 Tafeln ${ }^{5}$. Sie sind nach dem Urteil Hans Fischers «ein unübertreffliches Muster einer barocken Darstellung» und bezeugen Füßlis überragende Erfindungsgabe ${ }^{6}$. Die Geburtstafel läßt sich damit nicht vergleichen. Immerhin wurde sie von dem Vierundzwanzigjährigen exakt konstruiert und sauber ausgeführt. Als Vorbild mag dabei der Zürcher Regimentsspiegel von 1657 gedient haben: Mit 160 Scheiben und vielen Wappen stellte Hans Heinrich Schwyzer auf Tafeln, die zusammen einen Schrank bilden, die Regierungsämter und ihre Inhaber dar?

Das Ehegericht, dem Muralt den Schwangerschaftskalender verehrte, überwachte seit der Reformation die Sittenzucht im Stande Zürich. Von Amtes wegen ging es außerehelichen Vaterschaften nach, denn der Vater hatte einen angemessenen Beitrag an den Unterhalt des Kindes zu zahlen. Die Schwangere war überdies verpflichtet, ihren Zustand anzuzeigen, sei es dem Pfarrer, sei es unmittelbar dem Ehegericht ${ }^{8}$. Die Mitglieder von 1701 sind mit ihren Namen und Wappen im oberen Teil der Tafel aufgeführt ${ }^{9}$. Es sind dies: 
Als Praeses: Johann Ludwig Hirzel (1652-1710), Statthalter; in seinem Todesjahr wurde er Bürgermeister ${ }^{10}$.

Von Geistlichen: Hans Bernhard Werdmüller (1655-1707), Pfarrer bei Sankt Peter ${ }^{11}$; Hans Wilhelm Hoffmeister (1652-1715), Leutpriester beim Großmünster $^{12}$.

Von Räten (Mitgliedern des Kleinen Rates): Beat Holzhalb (1638-1709), gewesener Landvogt der Grafschaft Kiburg, ein in Padua ausgebildeter Jurist und Poet ${ }^{13}$; Hans Heinrich Escher [vom Luchs] (1673-1724), gewesener Landvogt der Herrschaft Greifensee ${ }^{14}$.

Von Burgern (Mitgliedern des Großen Rates): Hans Heinrich Keller, gewesener Amtmann am Ötenbach ${ }^{15}$; Hans Felix Aberli († 1715), gewesener Kammerer des Großmünsterstifts ${ }^{16}$; Hans Wilhelm Wolff (1638-1710), gewesener Landvogt der Freiherrschaft Sax im Rheintal, von Beruf Glasmaler ${ }^{17}$.

Als Schreiber: Johannes Rahn (1655-1732)18.

Als Weibel: Meister Jost Vogel $(\dagger 1726)^{19}$.

Wieweit die Geburtstafel im Ehegericht Anwendung fand, ist nicht bekannt. In medizinischen Fragen stand ihm die «Kleine Gschau», der der Stadtarzt und der Stadtschnittarzt angehörten, als Sachverständigengremium zu Gebote ${ }^{20}$. Immerhin wurde die von Muralt verfaßte Gebrauchsanweisung zu dem Instrument einem aus Privatbesitz stammenden Exemplar der Ehegerichtsordnung von 1697/ 1698 am Schluß als Abschrift beigefügt ${ }^{21}$. In den amtlichen Exemplaren der Ordnung von 1697/1698 und 1719 fehlt sie ${ }^{22}$.

\section{Muralts Gebrauchsanweisung}

Die «Beschribung der geburts-Taffellen, Auß welicher seye gewüße zeith der empfängnus und geburt eines kinds in muter leib zu verstehen» (siehe Anhang) wird beiläufig von Marianne Bernet in ihrer Dissertation Der Beizug von gerichtlichen Sachverständigen im alten Zürich, 1967, S.158, erwähnt. Die nur als Kopie erhaltene Widmungsschrift ist von Muralt auf den 5. Dezember 1701 datiert. Sie zerfällt in eine Beschreibung und in eine Weisung zum «gebrauch des Instruments». Daraus geht hervor, daß als Beginn der Schwangerschaft der erste Tag nach dem Aufhören der Menstruation angenommen wird. «Zum Exempel wann

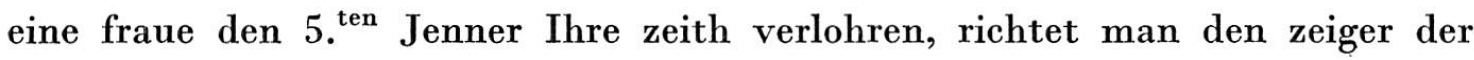
empfängnus auf den sechsten grad des ersten unbeweglichen rads, und kehrt daß ander rad in den ersten monds-monath der empfängnus auf den sechsten grad des ersten unbeweglichen rads, deßgleichen daß drite in dem sechsten grad der schwangerschafft und daß vierte in den ersten quadragenarium, so wird man also 
bald sechen daß im anfang des Julij das halbe Jahr..., die geburth aber in den anfang des weinmonath [etwa 11. Oktober] ... kommen werde» (Anhang S. 14). «Wann aber eine frauen nicht weißt wan sie den anfang bekommen, oder Ihre reinigung bestanden, frag man sie, wan sich daß kind zu erst beweget habe, und richtet den halb-Jahr zeiger auf die selbe zeith in allen Cirklen, so weißt man alle zeith wann die empfängnus geschechen und daß kind gebohren werden solle» (Anhang S. 15).

Während die Zeiger zur Bestimmung von Anfang und Ende der Schwangerschaft dienen, erlauben die entsprechend eingestellten Scheiben die Beurteilung der kindlichen Entwicklung. Die Mondmonate (29 1/2 Tage) im zweiten und die vierzigtägigen Abschnitte im vierten Kreis entsprechen der Stadieneinteilung der Schwangerschaft in den hippokratischen Schriften (siehe unten). Muralt benützt die Kalendermonate des dritten Kreises, um am Schluß der Gebrauchsanweisung den Eherichtern die Stufen der fötalen Entwicklung zu erklären (Anhang S. 15 f.).

\section{Die Schwangerschaftsdauer}

Durch den konstanten Winkel zweier Zeiger gibt Muralts Kalender, ausgehend vom Ende der letzten Periode, die Dauer der Schwangerschaft mit 278 Tagen an. Damit wäre er auch heute noch benützbar, wenn auch mit der Einschränkung, «daß $2 / 3$ aller Kinder 3 Wochen um den errechneten Geburtstermin herum geboren werden ${ }^{23}$

Daß Muralt den Zeitpunkt der Geburt als genau definiert annimmt, ergibt sich aus der Gebrauchsanweisung. Nicht nur fehlt eine Angabe, die die Zeigergenauigkeit relativiert und den Termin als Zeitspanne aufzufassen erlaubte; vielmehr wird unbedenklich die Rückrechnung auf den Schwangerschaftsbeginn empfohlen. Wenn mehrere Männer « mit der frauenen zu schaffen gehabt, muß man eines ieden ersten beyschlaff erkundigen, den zeiger der empfäng $\langle\mathbf{n}\rangle$ us darauff richten, wann dann die geburth mit dem zeiger nit eintrifft muß man dem kind einen anderen vatter suchen» (Anhang S. 15). Ebenso zuversichtlich glaubt Muralt den Termin nach den ersten Kindsbewegungen mit dem Halbjahreszeiger bestimmen zu können.

In seinen gedruckten Schriften ist Muralt weniger präzis. Nach dem «Kinderund Hebammen-Büchlein» (1697) werden die ersten Kindsbewegungen «bald in 20 , bald in 15, 16, 17, 18 und mehr Wochen » verspürt ${ }^{24}$. Die Geburtszeit wird an mehreren Stellen verschieden definiert und auf das «End des 9 . Monats ${ }^{25}$ oder «gemeiniglich in den zehenden Monat» ${ }^{26}$ verlegt; das Kind bleibt «völlig neun Monat und offt darüber ${ }^{27} \mathrm{im}$ Mutterleib; «die längste Zeit der Geburt ist von 10 Monaten. ${ }^{28}$ 
Zu Muralts Zeit stand die Frage nach Norm und Grenzen der Schwangerschaftsdauer durchaus offen. Wie sich die Auseinandersetzung mit dem theoretisch und praktisch gleichermaßen wichtigen Problem entwickelte, soll im folgenden anzudeuten versucht werden ${ }^{29}$.

In den hippokratischen Schriften gelten das Ende des neunten und der zehnte Monat, an anderen Stellen der 280.Tag (siebenmal vierzig Tage) als Geburtstermin. Auch Siebenmonatskinder sind lebensfähig, die Achtmonatskinder aber sterben $^{30}$. Länger als zehn Monate dauernde Schwangerschaften sind ausgeschlossen, da die Ernährung des Fötus ungenügend wird und er gezwungen ist, ans Licht zu treten ${ }^{31}$. So gilt Hippokrates der Nachwelt als Stifter der Regel, daß die Schwangerschaft zeitlich beschränkt ist, und auch, trotz gewissen Schwierigkeiten, der Neunmonateregel. Diese fand für das christliche Abendland ihre Bestätigung lange Zeit darin, daß die heilige Anna mit Maria, Maria wiederum mit Jesus genau neun Monate schwanger ging, - in einer Annahme also, die sich auf die im 6. und 8. Jahrhundert entsprechend angesetzten kirchlichen Festtage stützte $^{32}$. Was noch Paulus Zacchias (1584-1659) ${ }^{33}$ als Beweis der vollkommenen Schwangerschaftsdauer diente, beleuchtet immerhin die Auffassung des Frühmittelalters, die bis heute die volkstümliche geblieben ist. Auch Eucharius Rößlin $(1513)^{34}$, Ambroise Paré (1510?-1590) ${ }^{35}$ und Jakob Rueff (1569) ${ }^{36}$ stehen in dieser Tradition. Für François Mauriceau (1637-1709) - Muralt hörte als Student in Paris seine Vorlesungen ${ }^{37}$ - sind neun Monate «le terme le plus ordinaire». Länger als zehn Monate dauernde Schwangerschaften konnte er nicht beobachten $^{38}$. Pierre Dionis $(\dagger 1718)$ hält nur den für einen aufgeklärten Chirurgen, der die Neunmonateregel als Naturgesetz anerkennt ${ }^{39}$.

Grundsätzlich verschieden ist die Auffassung, daß der Zeitpunkt der Geburt keiner bestimmten Regel unterworfen ist. Sie beruft sich auf den Satz von Aristoteles: «... einzig unter allen Geschöpfen der Mensch hat viele Tragzeiten, Kinder kommen mit sieben, acht, neun, höchstens zehn, ganz wenige auch mit elf Monaten. $~^{40}$ Dem pflichten Plinius der Jüngere ${ }^{41}$ und im 16. Jahrhundert neben anderen Horatius Augenius (1527-1603) bei ${ }^{42}$.

Der Arzt und Chirurg, der vor Gericht als Sachverständiger aufzutreten hatte, mußte bei Legitimitätsprozessen Stellung zur Schwangerschaftsdauer nehmen ${ }^{43}$. Gab es für den Geburtstermin eine bestimmte Regel, ließ sich auch der Zeitpunkt der Konzeption errechnen. Dann waren die Fälle zu früh geborener ehelicher und postumer Kinder leichter zu beurteilen. War der Geburtstermin nicht vorbestimmt, mußte vermehrt auf die Aussagen der Beteiligten, auf die Ehrbarkeit der Frau geachtet werden, sollte nicht der Leichtfertigkeit Vorschub geleistet werden. In beiden Fällen boten die Frühgeburten geringere Schwierigkeiten; sie 
waren mehr oder weniger reif; vor dem siebenten Monat galten die Kinder meist nicht als lebensfähig ${ }^{44}$. An den Spätgeburten dagegen schieden sich die Geister. Die damit zusammenhängenden Fragen werden deshalb von den gerichtsmedizinischen Autoren besonders ausführlich abgehandelt.

Battista Codronchi (Mitte des 16. Jahrhunderts) sieht mit anderen Autoren ein, daß verminderte Bildungsfähigkeit, weibliches Geschlecht des Fötus und Mängel verschiedener Art die Geburt leicht bis zum elften Monat hinauszögern können. Der Einwand der Alten, daß die Nahrung des Fötus beschränkt sei, bringt ihn auf die Kompromißformel, daß der von Hippokrates angegebene Termin des neunten oder zehnten Monats zwar häufiger sei, daß aber Ausnahmen vorkommen und die Geburt in die ersten Tage des elften Monats fallen könne ${ }^{45}$.

Fortunatus Fidelis (etwa 1550-1630) beansprucht für den Geburtstermin einen Zeitraum von einer gewissen Breite (dierum spatium, quod tamen in quadam latitudine positum sit), nämlich einen Teil des neunten und den größten Teil des zehnten Monats, also das siebente «dierum quadragenarium ».46 Eine aus langer Erfahrung gezogene Faustregel besagt, daß der Zeitpunkt der ersten Kindsbewegungen, mit drei multipliziert, den Tag der Geburt ergibt ${ }^{47}$. Den Beginn der Schwangerschaft nimmt Fidelis mit dem Ende der letzten Menstruation an. Alle vor dem Termin geborenen Kinder sind irgendwie unreif (aliqua ex parte semper esse imperfectos); dagegen ist es möglich, daß der Fötus wegen einer Hemmung der Natur (impedimento naturae) mehr Zeit als üblich zur vollkommenen Ausbildung braucht und sich die Schwangerschaft ungewöhnlich verlängert ${ }^{48}$.

Paulus Zacchias (1584-1659) stimmt, was den Termin betrifft, im wesentlichen mit Fidelis überein. Abweichungen interpretiert er aber strenger; sie sind für ihn pathologisch (non à natura, \& consequenter à morbo ${ }^{49}$.

Die Frage nach der Möglichkeit verlängerter Schwangerschaften war im 17.Jahrhundert umstritten; sie blieb es auch im 18.Jahrhundert. Bei gutem Leumund der Frau waren die medizinischen Fakultäten und die Gerichte geneigt, Elf- und Zwölfmonatskinder anzuerkennen ${ }^{50}$. Laurenz Heister (1683-1758) und die Fakultät von Helmstedt betrachteten 1721 ein nach 13 Monaten geborenes Kind als legitim ${ }^{51}$. 1764 erregte es in Frankreich Aufsehen, daß Antoine Louis (1723-1792) - sich auf die unveränderlichen Naturgesetze berufend einem Kind die Legitimität aberkannte; es war zehn Monate und 17 Tage nach dem Tod seines angeblichen Vaters zur Welt gekommen, der 76jährig anderthalb Monate schwer krank gelegen hatte ${ }^{52}$. Louis' Konsultation unterstützten vier weitere Chirurgen, ebenso der Arzt Michel-Philippe Bouvart (1717-1787) ${ }^{53}$. Dagegen erhoben sich der Geburtshelfer Jean Lebas (1717-1797) ${ }^{54}$, der Anatom und Physiologe Exupère-Joseph Bertin (1712-1781) ${ }^{55}$, allen voran der bekannte Arzt 
Antoine Petit (1722-1794) ${ }^{56}$ mit eigenen Streitschriften. Sie unterschieden drei Kategorien von Geburten, die natürlichen nach einer Schwangerschaft von neun Monaten und einigen Tagen, die seltenen nach sechs, sieben, acht und zehn Monaten, und die außerodentlichen nach elf und mehr Monaten. Daraus folgt: «Donc l'accouchement n'a point de terme fixe; donc il est possible, quoique extraordinaire, qu'une femme accouche d'un enfant à onze mois \& au-delà. » ${ }^{57}$

Albrecht von Haller (1708-1777) gestattet in seiner gerichtlich-medizinischen Vorlesung Abweichungen von lediglich ein bis zwei Wochen von der Normaldauer von 39 Wochen. Elf-, Zwölf- und Dreizehnmonatsgeburten sind «wider alle Geseze der Natur». ${ }^{58}$ Als Physiologe nahm Haller während der Pariser Kontroverse zu unserem Problem Stellung. Es sei klar, daß die Geburtszeit nicht ohne eine gewisse Breite sein könne. Übermächtige innere und äußere Ursachen könnten sie beeinflussen. Vor der 39. Woche geborene Kinder weisen auf alle Fälle Zeichen der Unreife auf. Viele verlängerte Schwangerschaften sind unglaubwürdig (unsicherer Konzeptionstermin, absichtliche Täuschung); ganz unmöglich sind sie offenbar nicht ${ }^{59}$.

Für die Verfechter verlängerter Schwangerschaften löst der Fötus beim Erreichen einer gewissen Größe die Geburt aus, was die Gegner, so Antoine Louis ${ }^{60}$, bestreiten. Bei François-Emmanuel Fodéré (1764-1835) verzögert eine langsame Entwicklung auch den Geburtstermin. Das übertragene Kind sei entgegen den Ansichten einiger Autoren und des gemeinen Volkes nicht entsprechend schwerer und weiter gereift, sondern im Gegenteil geschwächt, wenn auch von eigentümlich ältlichem Aussehen. Als «non plus ultra» anerkennt Fodéré zwei Monate zu den üblichen neun ${ }^{61}$. Demgegenüber lenkten im 19. Jahrhundert deutsche Geburtshelfer und Physiologen die Aufmerksamkeit vermehrt auf die Zusammenhänge mit dem Menstruationszyklus. Nach Friedrich Benjamin Osiander (1759-1822) ereignet sich die Geburt «mit der Zeit, wo das Monatliche, nachdem es neunmal ausgeblieben ist, zum 10 ten $\mathrm{Mal}$ wieder erscheint ...» Osiander begrüßt, daß gerichtliche Ärzte nicht mehr starr an der Vierzigwochenregel festhalten, sondern dieses Vorurteil ablegen ${ }^{62}$. Arnold Adolph Berthold (1803-1861) präzisiert : «Daß nach der Verschiedenheit der Menstruationsperioden die Schwangerschaftsdauer verschieden ist, und daß in den regelmäßigen Fällen die Geburt eintritt, wenn sich der Eierstock zur zum 10. male wiederkehrenden Menstruation vorbereitet ... » ${ }^{63}$ Paul Loewenhardt (geb. 1862) glaubte gefunden zu haben, « daß die Entfernung des Eintritts der vorletzten Periode von dem Eintritt der letzten Zeit den zehnten Theil der Schwangerschaftsdauer von da ab bis zur Geburt ausmacht ... ${ }^{64}$ Dies bestritt der Zürcher Doktorand Max Hasler als Schüler Ferdinand Frankenhäusers (1832-1894): «Unter absolut normalen Verhältnissen ist 
die Dauer jeder Schwangerschaft dieselbe.» «Eine Menge zufälliger, durch äußere Umstände wie durch Verschiedenartigkeit der Individualität jeder einzelnen schwangeren Frau bedingter Einflüsse» können allerdings die Geburt «um Tage, ja einzelne Wochen früher oder später herbeiführen ». ${ }^{65}$

Was die Statistiker umständlich bewiesen ${ }^{66}$, hatte man in der praktischen Geburtshilfe immer gewußt. Zur einfachen Berechnung des Zeitabstandes von 280 Tagen hatte sich von 1833 an die Regel von Franz Karl Nägele (1778-1851) durchgesetzt; sie lautet: "Man rechnet von dem Tage an, wo die Frau ihre Reinigung zum letzten Male gehabt, 3 ganze Monate zurück und zählt dann 7 Tage hinzu; der so gefundene Tag ist alsdann derjenige, an welchem die Niederkunft zu erwarten ist. ${ }^{67}$

130 Jahre später wird die Nägelesche Regel immer noch empfohlen ${ }^{68}$. Inzwischen hat die Hormonforschung besonders in unserem Jahrhundert grundlegend neue Einsicht in die gegenseitige Abhängigkeit von Uterus, Ovar und Hypophyse gewonnen. Hermann Knaus (*1892) konnte 1929 den Zeitpunkt der Ovulation auf den 15.Tag vor der nächsten zu erwartenden Monatsblutung festlegen ${ }^{69}$. Knaus forderte deshalb 1970, die Nägelesche Regel endlich fallenzulassen. Er empfiehlt, mit Hilfe des Menstruationskalenders die durchschnittliche Zykluslänge der einzelnen Frau zu berechnen und anschließend den Tag der Ovulation bzw. der Konzeption (den 15. vor der nächsten zu erwartenden Periode) zu ermitteln. Der Geburtstermin liegt 273 Tage oder 39 Wochen später. Kinder mit allen Reifezeichen sind vom 250. Tage post ovulationem zu erwarten. Alle länger als 39 Wochen dauernden Schwangerschaften sind als übertragen zu betrachten. 64 der 139 von Knaus bis 1970 beobachteten Schwangerschaften endeten vor dem mit der neuen Methode berechneten Termin. In neun Fällen trat die Geburt verspätet am 280. bis 300. Tag post conceptionem ein, entsprechend dem 293. bis 307.Tag nach der letzten Menstruation ${ }^{70}$.

Allen besprochenen Autoren ist gemeinsam, daß sie sich um das tiefere Verständnis eines fundamentalen biologischen Geschehens bemühen. Während der eine jede Gesetzmäßigkeit leugnet, sieht der andere eine feste Regel als Grundlage; die Abweichungen sind für ihn Artefakte oder die Folge übermächtiger

Abb.1. Schwangerschaftskalender («Geburts-Tafel»), 1701 vom Zürcher Stadtarzt Johannes von Muralt (1645-1733) dem Ehegericht Zürich geschenkt. Oben die Namen und Wappen der Eherichter, unten die Kalendarien zur Bestimmung des Zeitpunkts der Geburt. Medizinhistorische Sammlung der Universität Zürich 


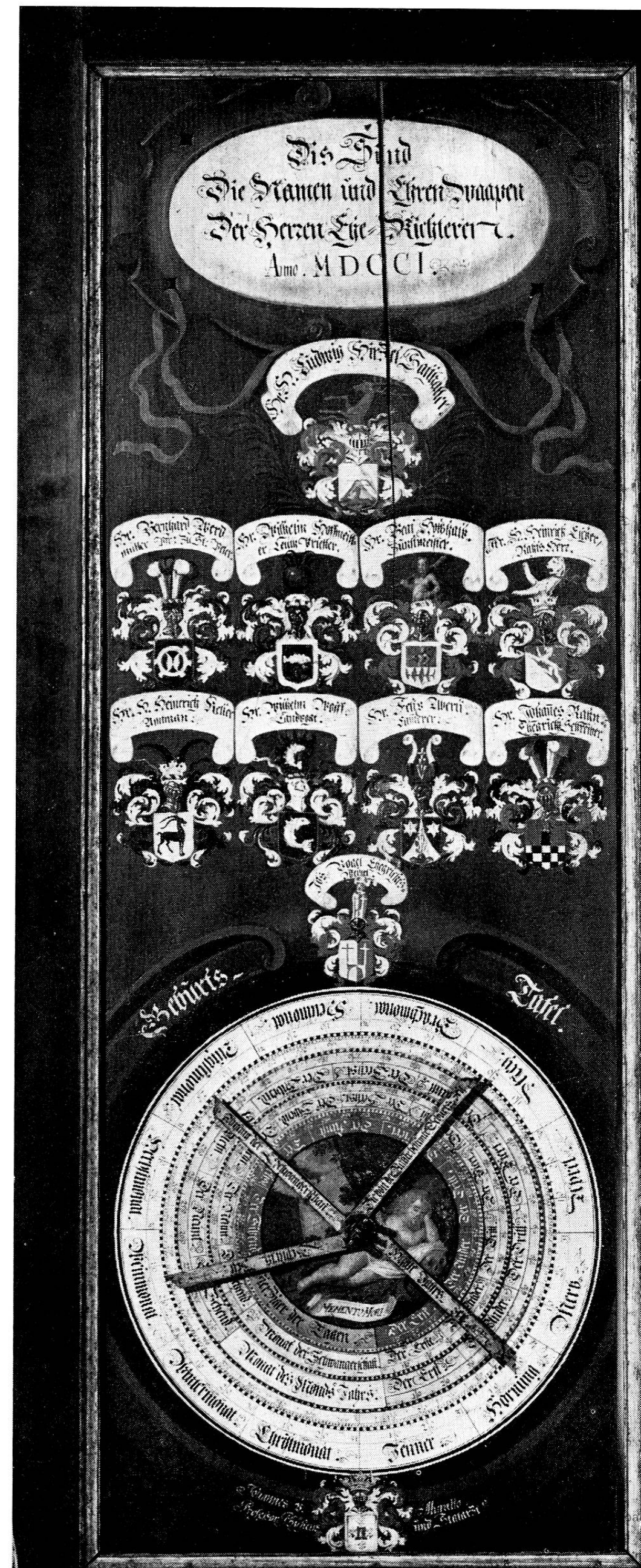




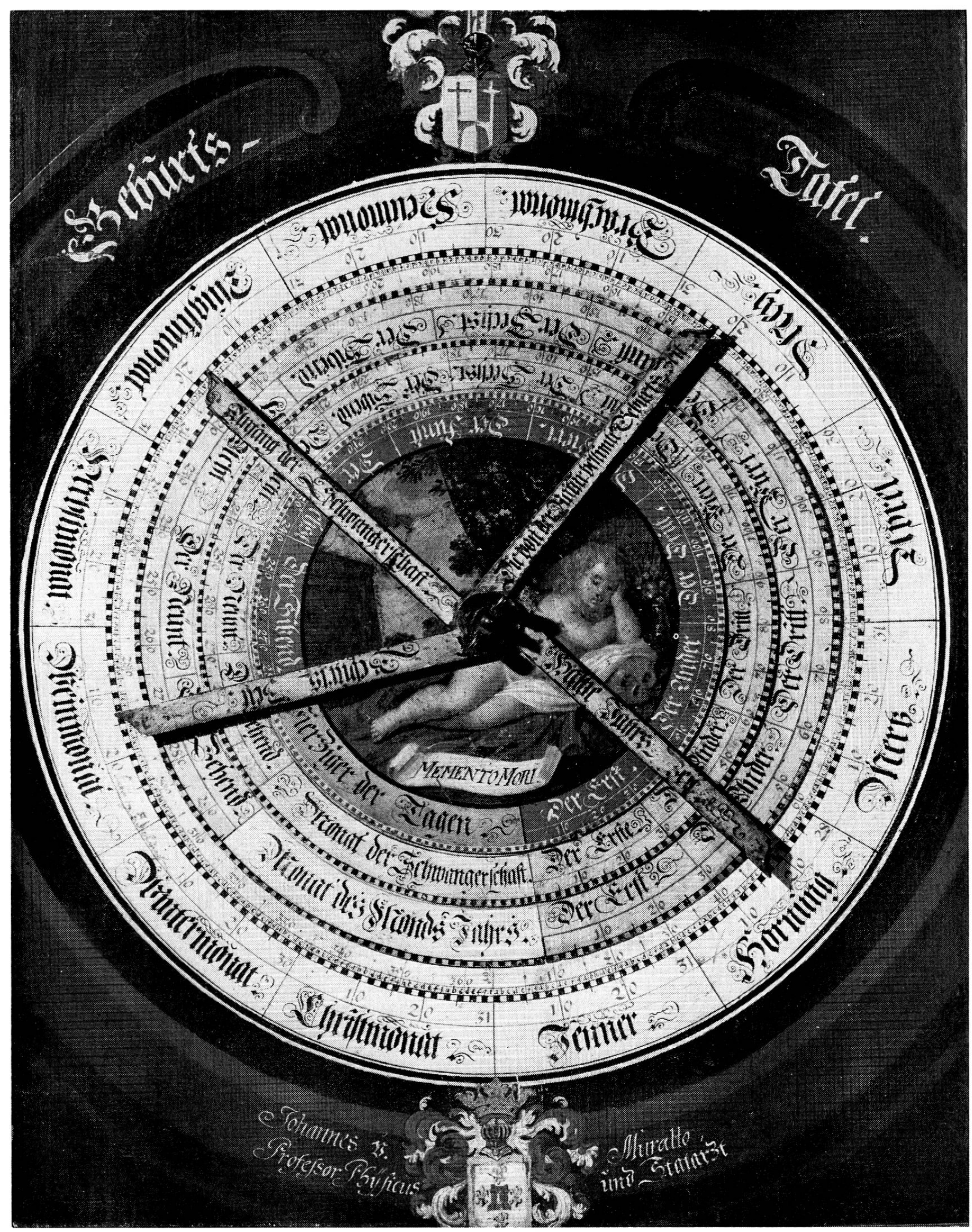

Abb. 2. Muralts «Geburts-Tafel» mit vier Kalendarien (Sonnenjahr, zehn Mondmonate, zehn Schwangerschaftsmonate, sieben vierzigtägige Abschnitte). Die drei inneren Kreise sind auf drei drehbare Scheiben gemalt. Mit drei fest miteinander verbundenen Zeigern werden der «Anfang der Schwangerschaft», die «Halbe Jahrs-Zeit» und «Die von der Natur bestimte Geburts-Zeit», der 278. Tag, eingestellt. Der vierte Zeiger (« Geburts-Zeit») dient zur Markierung allfälliger Abweichungen 


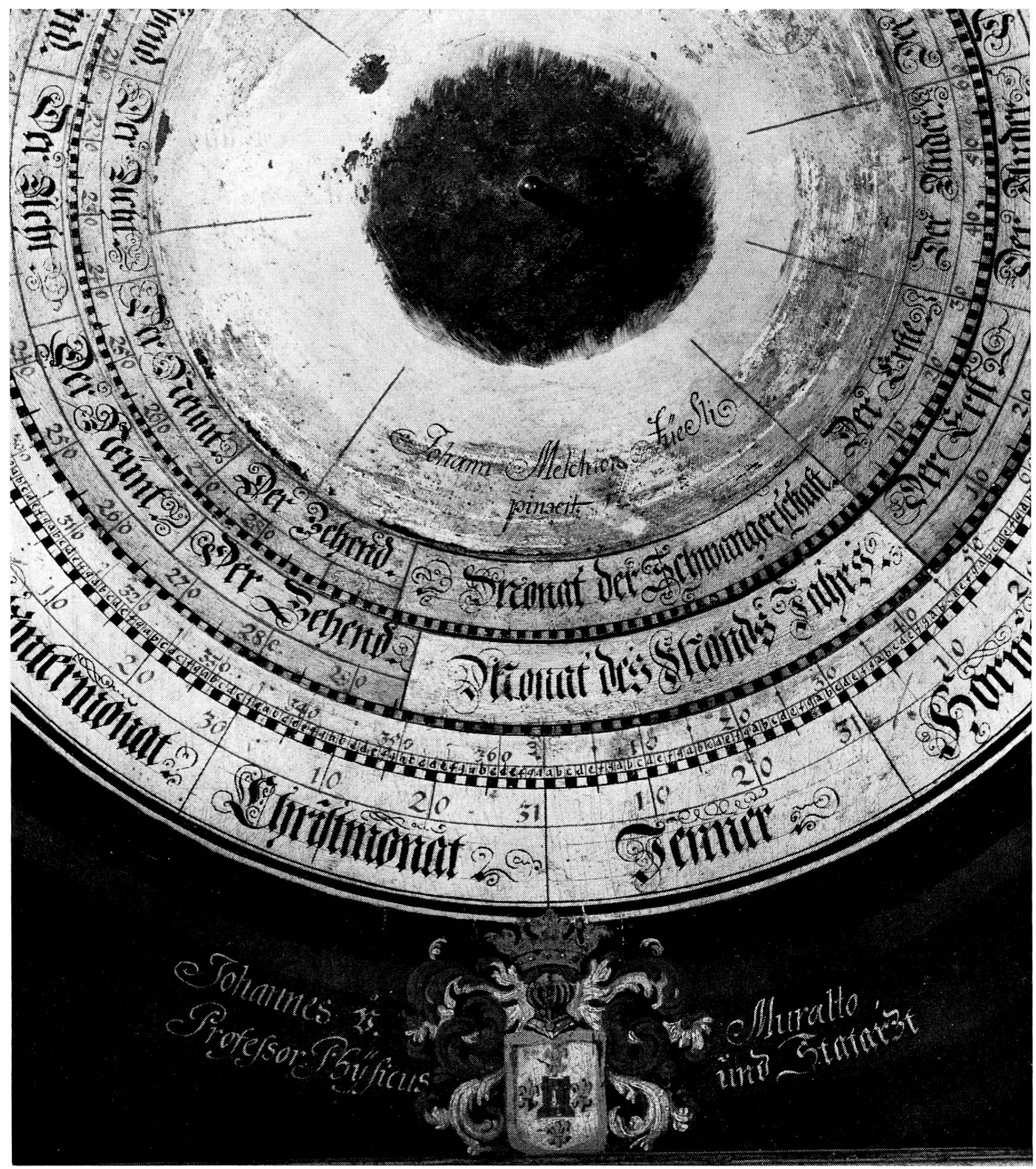

$A b b$. 3. Muralts «Geburts-Tafel» nach dem Abnehmen der obersten Scheibe: Signatur des Malers Johann Melchior Füßli (1677-1726) von Zürich 


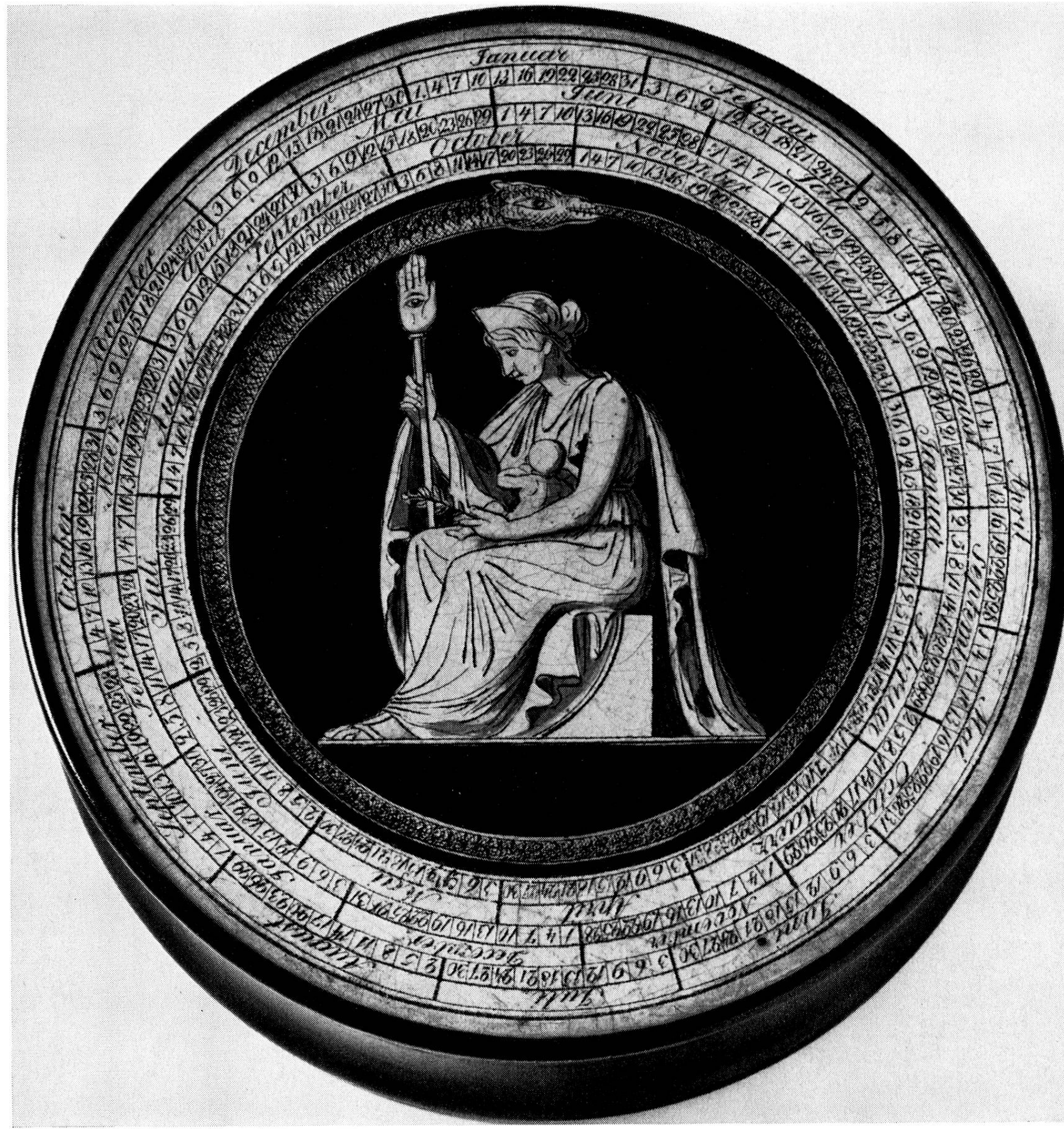

$A b b$. 4. Schwangerschaftskalender auf einer Schnupftabakdose, erfunden 1826 von Karl Alexander Ferdinand Kluge (1782-1844), Geburtshelfer in Berlin. Die Juno Lucina als Beschützerin der Gebärenden wird umrahmt von der Schlange und drei Kalendarien, die dem Anfang, der Mitte und dem Ende der Schwangerschaft (280.Tag) entsprechen. Medizinhistorische Sammlung der Universität Zürich. Fotos: Paul Scheidegger, Zürich 
störender Ursachen. Die statistische Methode, ein Kind des 19. Jahrhunderts, findet sich damit $a b$, daß der Einzelfall nicht genau vorauszuberechnen ist. In der Praxis behilft man sich von jeher mit Faustregeln oder einfachen Hilfsmitteln, den Schwangerschaftskalendern.

\section{Notizen zur Geschichte der Schwangerschaftskalender}

Muralts «Geburts-Tafel» ist für die Zeit vor 1800 der einzige mir bekannt gewordene Schwangerschaftskalender. 1820 setzt der in Dresden wirkende Naturphilosoph und Geburtshelfer Carl Gustav Carus (1789-1869) - offenbar als einer der ersten - drei parallele Kalenderkolonnen miteinander in Beziehung. Sie entsprechen dem mutmaßlichen Tag der Konzeption, der Mitte der Schwangerschaft (erste Kindsbewegungen) und dem Geburtstermin (280.Tag) ${ }^{71}$. Adam Elias von Siebold (1775-1828) beschreibt 1826 einen Schwangerschaftskalender, der auf einer Schnupftabakdose angebracht ist, erfunden von Karl Alexander Ferdinand Kluge (1782-1844), dem Direktor der Gebäranstalt der Berliner Charité ${ }^{72}$. Kluges «Lucinendose » ${ }^{73}$ (Abb. 4) trägt auf der Vorderseite des Dekkels das Bild der Juno Lucina und drei konzentrische Kalendarien zur Bestimmung der Mitte und des Endes der Schwangerschaft (280.Tag). Auf der Innenseite des Deckels werden für die einzelnen Monate die Charakteristiken der menschlichen Frucht, auf der Außenseite des Bodens die Erscheinungen am mütterlichen Körper aufgeführt. - Ein Periodoskop wurde um 1850 von William Tyler Smith (1815-1873) entwickelt ${ }^{74}$. Mehr oder weniger komplizierte Tabellen stammen von Bernhard Sigmund Schultze (1828-1924) ${ }^{75}$, Alexandre Lacassagne (1843-1924) ${ }^{76}$, Pierre-Victor-Alfred Auvard (geb.1855) ${ }^{77}$ und Smith aus Warschau $^{78}$. Eine im American Journal of Obstetrics 1889 erschienene Tafel stimmt fast genau mit dem Klugeschen Kalendarium überein ${ }^{79}$.

\section{Anhang: Johannes von Muralts Beschreibung der «Geburts-Tafel»}

Staatsarchiv Zürich, B III 62 a ; Abschrift

\section{[1] Beschribung der geburts -Taffellen}

Auß welicher seye gewüße zeith der empfängnus und geburt eines kinds in muter leib zu verstehen.

Diße taffellen bestehet auß vier räderen, welche in der mite an einem Nagel vest, deren drey rund-umb denselben beweglich: Ein iedes diser räderen hat nebent gemeiner abtheilung auch seine eigentliche bedeutung und gebrauch, fürnemlich den Ärtzten zu wüßen Nothwendig. 
1. - Daß erste, und gröste unbewegliche Rad welches die anderen einschliesßt, wird in 365. tag oder 12 theil (gleich wie daß Jullianische Jahr in 12 monath abgetheilt ist:) underscheiden.

2. - Daß andere begreifft in sich die mondsmonath, deren ieder $29 \frac{1}{2}$ grad behaltet, nach den tagen eines monds-monath,

[2] 3. - Daß drite wird getheilt in die Monath der schwangerschafft, deren ieder 30 tag behelt und zeiget daß wachsthum der kinderen in muter leib von einem Monath zu dem anderen.

4. - Daß vierte ist in 40 grad der tagen, daß ist in 7 gleiche portionen abgetheilt deren iede 40, theil hat.

Über diße 4 Räder ist ein dreyfacher vester zeiger gesetzt, welcher selbige in 2 gleiche theil theilt, und auff der einten seithen den tag der empfängnus, auf der anderen aber daß halbe Jahr von der empfängnus oder bewegung deß kinds andeutet, und endtlich ist noch ein anderer zeiger auf der driten seithen, welcher den tag der geburth vorstellet, der vierte zeiger ist beweglich und zeiget an die abweichung von der geburthstund, deren Exempel hernacher zu finden.

\section{[3] gebrauch des Instruments}

Wann der Eherichter oder Medicus wüsßen wil, wie lang daß kind in muter leib gelegen, muß er sich wo möglich, informmieren, wann der frauen die monaths Reinigung bestanden, schließt dann volgenden tag darauff ungefehrd seye die empfangnus beschechen, kehret den zeiger alßdann auf des anderen Rads anzeigten tag, und richtet daß drite rad gleich dem anderen wie auch daß vierte rad. Zum Exempel wann eine fraue den $5 .{ }^{\text {ten }}$ Jenner Ihre zeith verlohren, richtet man den zeiger der empfängnus auf den sechsten grad des ersten unbeweglichen rads, und kehrt daß ander rad in den ersten monds-monath der empfängnus auf den sechsten grad des ersten unbeweglichen rads, [und kehrt daß ander Rad in den ersten monds-monath der empfängnus] ${ }^{80}$

[4] deßgleichen daß drite in dem sechsten grad der schwangerschafft und daß vierte in den ersten quadragenarium, so wird man also bald sechen daß im anfang des Julij das halbe Jahr, der anfang des $7^{\text {ten }}$ monds-monath, der $7 .^{\text {te }}$ monath der schwangerschafft, und der 5 . $^{\text {te }}$ quadragen $\langle a\rangle$ rius des ineren Cirkels bedeütet werde, die geburth aber in den anfang des weinmonath in anfang des zehenden der schwangerschafft und zu end des sibenden quadragenarij kommen werde; Je nach dem nun der bewegliche kleine zeiger auf den tag der hochzeith gerichtet ist, weißt der Eherichter also bald, wann der früezeitige beyschlaff geschechen, 
und wie vill tag daß kind zufrüe kommen seye, alß zum Exempel so ein hochzeith tag gehalten worden were den $5 .{ }^{\text {ten }}$ Jenner und die kindsgeburth geschechen

[5] den 29. ${ }^{\text {ten }}$ Julij selbigen Jahrs, so stelle die Regel des anfangs der schwangerschafft auf den volgenden tag nach der hochzeith alß den $6 .{ }^{\text {ten }}$ Jenner, die bewegliche Regel aber auf den $29^{\text {ten }}$ Julij alß geburthstag bey disem finde den buchstaben $G^{81}$ danachen zelle ich alle $G$, biß ich komme auff [?] den rechten von der Nattur bestimten geburths tag, den mir die Regul weißet, finde biß dahin an der anzahl 10. sagte hiemit, daß das kind umb sovil wochen zu früe worden seye, wann aber eine frauen nicht weißt wan sie den anfang bekommen, oder Ihre reinigung bestanden, frag man sie, wan sich daß kind zu erst beweget habe, und richtet den halb-Jahr zeiger auf die selbe zeith in allen Cirklen, so weißt man alle zeith wann die empfängnus geschechen und daß kind gebohren werden solle; kan man aber nichts alß die geburth in

[6] erfahrung bringen, so richtet man den geburtszeiger auf selbigen tag in allen vier Cirklen, da dann vollkommen herauß kommen wird die zeith der empfannus, und der geburth.

So man nicht eigentlich weißt, wer des kinds vatter ist, oder Ihren etliche mit der frauenen zu schaffen gehabt, muß man eines ieden ersten beyschlaff erkundigen, den zeiger der empfäng $\langle\mathbf{n}\rangle$ us darauff richten, wann dann die geburth mit dem zeiger nit eintrifft muß man dem kind einen anderen vatter suchen.

Mann weißt auch auß dem driten [g]rad, wie daß kind in muter leib biß in anfang des zehenden monaths wachst, ob solches außgetragen oder nicht, dan im ersten monath in der gebährmuter nur ein trüebes Eylin, mit einem zarten Stychlin ${ }^{82}$ in größe einer haßelnusß, von

[7] unden mit fleisch faßeren umbschloßen gefunden wird, im anderen und driten monath, wird auß disem Stychlin die zerspreitete Nabelschnur, und wird daß Ey einer Nusß groß, man sihet auch darin ein gar artlich formmiertes beingerüst, daran der kopf vast so groß alß der gantze leib, und lebt in stetter bewegung, im vierten monath kent man alle glidmaßen, und ist daß kind fast eines vierlings lang, allezeith in einem solchen Ey mit geväßen eingeschlosßen, die Nabelschnur tringt durch den vast ofnen bauch in des kinds leberen; den $5 .^{\text {ten }}$ und $6{ }^{\text {ten }}$ monath formmierend sich alle theile, so zur speißmachung und blutkochung gedeyen, und ist die empfindung des kinds durch seine bewegung augenscheinlich, den sibenden monath beschließt sich die Nabelschnur im bauch vest, und fangt daß kind follig durch den mund sein eigen nahr- 
[8] ung zu sich zu nemmen ${ }^{83}$, und komt also biß zum end des neünten monaths in sein vollkommenheit, und wird der Nachgeburth sammt dem Stychlin oder Nabelschnur gantz steiff, so das die Nattur durch die kindswee selbige abstoßen, die gebährmuter sich öffnen, und daß kind gebohren werden musß.

Den $5 .^{\text {ten }}$ Decemb. A. ${ }^{\circ} 1701$.
Offerieret in gehorsam und underthänigkeit Eines Ehrsamen Ehegricht

Johannes von Muraalt ${ }^{84}$ Doctor Med. et Chir. StattArtzet

Anmerkungen

${ }^{1}$ Medizinhistorische Sammlung der Universität Zürich, Inventarnummer 387; Vorbesitzer: Frauenklinik Zürich (1919). Vgl. dazu: Conrad Brunner und Wilhelm von Muralt, Aus den Briefen hervorragender Schweizer Ärzte des 17. Jahrhunderts, Basel 1919, S. 262 und Abb. Nr. 9; Marianne Bernet, Der Beizug von gerichtlichen Sachverständigen im alten Zürich, Zürich 1967 (Zürcher Beiträge zur Rechtswissenschaft, N.F., Heft 275), S.158, 206, Tafel IV; Erwin H.Ackerknecht, Die Zürcher Schwangerschaftsuhr, in: Ursula Isler-Hungerbühler (Hrsg.), Zürcher Album, Wabern 1970, S. $76 \mathrm{f}$.

${ }^{2}$ [J. Finsler, Über J. von Muralt,] 55. Neujahrsblatt ... auf das Neujahr 1833 ... der Gesellschaft auf der Chorherrenstube, Zürich 1832 (mit Bibliographie); Conrad Meyer-Ahrens, Die Arztfamilie von Muralt, insbesondere Joh. von Muralt, Arzt in Zürich, in: Schweizerische Zeitschrift für Heilkunde, Bd. 1, 1862, S. 268-289, 423-436; Bd. 2, 1863, S. 25-47; Brunner/von Muralt (Anm. 1), S. 60-62, 227-273; Otto Obschlager, Der Zürcher Stadtarzt Joh. von Muralt (1645-1733) und der medizinische Aberglaube seiner Zeit, Diss. med. dent., Zürich 1926; Bernhard Peyer, Die biologischen Arbeiten des Arztes Johannes von Muralt, 1645-1733, Thayngen 1946 (mit ausführlicher Bibliographie); Emil Usteri, Johannes von Muralts Kampf um die Einführung der Anatomie in Zürich, in: Zürcher Taschenbuch 1977, Zürich 1976, S. 29-37; J.H. Wolf, in: Dictionary of Scientific Biography, Bd. XV (Supplement I), New York 1978, S. $428 \mathrm{f}$.

${ }^{3}$ Möglicherweise sind 280 Tage gemeint; siehe unten.

${ }^{4}$ Allgemeines Künstler-Lexicon ..., Drittes Supplement ..., Zürich 1777, S. 79f.; Carl Brun (Hrsg.), Schweizerisches Künstler-Lexikon, Bd. I, Frauenfeld 1905, S. 524; Ulrich Thieme (Hrsg.), Allgemeines Lexikon der bildenden Künste ..., Bd.12, Leipzig 1916, S. 571 f.

${ }^{5}$ Johann Jacob Scheuchzer, Kupfer-Bibel ..., 4. Bde., Augspurg und Ulm 1731-1735; Bd. 1, Vorbericht, unpag.

${ }^{6}$ Hans Fischer, Johann Jakob Scheuchzer ..., Naturforscher und Arzt, Zürich 1973 (Neujahrsblatt der Naturforschenden Gesellschaft in Zürich, 175.Stück), S.106-108.

7 Rudolf Schnyder, Zürcher Staatsaltertümer ..., Bern 1975 (Aus dem Schweizerischen Landesmuseum 34), S. 14 und Abb. 2. Den Hinweis darauf verdanke ich Herrn Prof. Dr. Werner G.Zimmermann, Zürich.

${ }^{8}$ Bernet (Anm.1), S.145-159; Ida Bischof, Die Rechtsstellung der außerehelichen Kinder nach den zürcherischen Rechtsquellen, Diss. jur., Zürich 1931, S. 16-46. 
9 Übereinstimmend mit dem Urteilsbuch des Ehegerichts 1701, Staatsarchiv Zürich, YY 1 189, Titelblatt. Eine Übergabe des Kalenders fand ich nicht erwähnt.

${ }^{10}$ Historisch-biographisches Lexikon der Schweiz [HBLS], 7 Bde., 1 Supplementbd., Neuenburg 1921-1934; Bd. 4, S. 233.

${ }^{11}$ Hans Jacob Leu, Allgemeines Helvetisches ... Lexicon ..., 20 Theile, Zürich 1747-1765; Th.19, S. 319. Hans Jakob Holzhalb, Supplement zu dem allgemeinen helvetisch-eidgenössischen ... Lexicon ..., 6 Theile, Zug 1786-1795; Th. 6, S. 362.

12 HBLS, Bd. 4, S. 266.

${ }^{13}$ HBLS, Bd. 4, S. 281.

${ }^{14}$ Leu (Anm. 11), Th. 6, S. 433.

${ }^{15}$ Leu (Anm. 11), Th. 11, S. 66.

${ }^{16}$ Holzhalb (Anm. 11), Th. 1, S. 1.

17 HBLS, Bd. 7, S. 584.

18 HBLS, Bd. 5, S. 520.

${ }^{19}$ Holzhalb (Anm. 11), Th. 6, S. 213.

${ }^{20}$ Bernet (Anm. 1), S. 157f.; 1734 erkundigte sich das Ehegericht bei der «Kleinen Gschau » nach der Möglichkeit einer 47 wöchigen Schwangerschaft, 1792 nach einer 45 wöchigen.

${ }^{21}$ Staatsarchiv Zürich, B III $62 \mathrm{a}$, "Geschenk von Frl. Jenny und Anna von Steiner, Zürich, 6. Mai 1940 ». Die «Beschribung» findet sich am Schluß des Bandes, anschließend an das Register; ohne Originalpaginierung. Siehe den Anhang S. 13-16 oben.

${ }^{22}$ Staatsarchiv Zürich, B III 62 (ca. 1531-1698), B III 63 (1719).

${ }^{23}$ Willibald Pschyrembel, Praktische Geburtshilfe für Studierende und Ärzte, 12. und 13. Aufl., Berlin 1967, S. 16-18.

${ }^{24}$ Johannes von Muralt, Kinder- und Hebammen-Büchlein ..., Basel 1697, S. 5. - Der Halbjahreszeiger entspricht dagegen der 26. Woche.

25 Johannes von Muralt, Chirurgische Schrifften ..., Basel 1691, S. 514.

${ }^{26}$ Johannes von Muralt, Hippocrates helveticus ..., Basel 1716, S. 2.

${ }^{27}$ Muralt (Anm. 24), S. 5.

${ }^{28}$ Johannes von Muralt, Anatomisches Collegium ..., Nürnberg 1687, S. 199; Muralt fährt fort: «Welche Kinder nun nach dem Tod ihrer Vätter nach dieser Zeit [zehn Monate] gebohren werden, die soll man nicht für ehelich gezeugete Kinder halten.»

${ }^{29}$ Frau Prof. Dr. Esther Fischer-Homberger verdanke ich zum Folgenden wichtige Ángaben und Hinweise; insbesondere ließ sie mich Einsicht nehmen in das Kapitel «Schwangerschaftsdauer» der Vorlesung "Geschichte der Gerichtsmedizin», 1975.

${ }^{30}$ E. Littré (Ed.), Euvres complètes d'Hippocrate .., 10 Bde., Paris 1839-1861; Bd. 7, S. 437-453 (Du foetus de sept mois), S. 453-461 (Du foetus de huit mois). Gerechnet wird in Mondmonaten zu 291/2 Tagen. Vgl. dazu: H[einrich] Fasbender, Entwickelungslehre, Geburtshülfe und Gynäkologie in den hippokratischen Schriften, Stuttgart 1897, S. 102-106.

${ }^{31}$ Littré (Anm. 30), Bd. 7, S. 535 (De la nature de l'enfant).

32 8. Dezember Mariae Empfängnis, 8. September Mariae Geburt; 25. März Mariae Verkündigung, 25.Dezember Christi Geburt. Lexikon für Theologie und Kirche, Bd. 7, Freiburg 1962, Sp. 65-69; Bd.10 (1965), Sp. 984f.

${ }^{33}$ Paulus Zacchias, Quaestionum medico-legalium tomus primus, Lugduni 1726 (erstmals erschienen 1621-1650), S. 45. 
${ }^{34}$ Eucharius Rößlin, Der Swangern Frauwen und hebammen Rosegarten, Einführung von Huldrych M.Koelbing, Dietikon 1976 (Faksimile der Ausgabe Straßburg 1513), Kap.2, unpag.

${ }^{35}$ J.-F.Malgaigne (Ed.), CEuvres complètes d'Ambroise Paré ..., Bd. 2, Paris 1840, S. 623 (1551).

${ }^{36}$ Jacob Rueff, Ein schön lustig Trostbüchle von den empfengknussen und geburten der menschen ..., Zürich 1569, S. XVI (Knaben im 9., Mädchen «gemeinklich» im 10. Monat).

${ }^{37}$ Meyer-Ahrens (Anm. 2), S. 277.

${ }^{38}$ François Mauriceau, Traité des maladies des femmes grosses, et de celles qui sont accouchées..., Cinquième édition, Bd. I, Paris 1712, S. 201-206; idem, Observations sur la grossesse et l'accouchement des femmes..., Paris 1715, S. 461.

${ }^{39}$ [Pierre E.] Dionis, Traité general des Accouchemens..., Paris 1724, S. 198.

${ }^{40}$ Paul Gohlke (Hrsg.), Aristoteles, Tierkunde, Zweite Auflage, Paderborn 1957, S. 311 (Buch VI, Kap. 4).

${ }^{41}$ H.Rackham (Ed.), Pliny, Natural History, Bd. II, Cambridge, Mass./London 1969 (The Loeb Classical Library 352), S. 531 (VII. Buch, Kap. V.).

${ }^{42}$ Horatius Augenius, De hominis partu libri II (1579); eine Zusammenfassung davon verdanke ich Frau Prof.Dr. Esther Fischer-Homberger.

${ }^{43}$ Vorlesung von Frau Prof.Dr.Esther Fischer-Homberger (Anm. 29); Erwin H.Ackerknecht, History of Legal Medicine, in Ciba Symposia, Bd. 11, 1950-1951, Nr. 7, S.12861304.

${ }^{44}$ Nach Hippokrates (Anm. 30) und Aristoteles (Anm. 40). Ausnahmen kamen auch hier vor; vgl. die Zusammenstellung bei Haller, Elementa (Anm. 59), S. 421-425.

${ }^{45}$ Battista Codronchi, Methodus testificandi ..., Francofurti 1597, S. 201-211.

${ }^{46}$ Fortunatus Fidelis, De relationibus medicorum libri quatuor ..., ed. Paulus Ammann, Lipsiae 1674, S. 451-458.

${ }^{47}$ Fidelis (Anm. 46), S. 457. Die Regel stammt aus dem Corpus hippocraticum (Littré [Anm. 30], Bd. 5, S. 345; $6^{\mathrm{e}}$ livre des Epidémies, $8^{\mathrm{e}}$ section).

48 Fidelis (Anm. 46), S. 463-486.

${ }^{49}$ Zacchias (Anm. 33), S. 23-47; Zitat S. 29.

${ }^{50}$ So führt Fodéré (Anm. 61, S. 114f.) elf Fälle an, die von 1578 bis 1779 in Frankreich beurteilt wurden. Sechsmal wurde das Kind als legitim anerkannt (zehn- bis zwölfmonatige Schwangerschaften).

${ }^{51}$ Laurentius Heister [Praeses] und Joannes Gerardus Wagner, Dissertatio medico-forensis qua partus tredecimestris pro legitimo habitus proponitur et simul partui nullum certum tempus in universum tribui posse ..., 1727, Editio altera, Helmstadii 1753.

${ }^{52}$ [Antoine] Louis, Mémoire contre la légitimité des naissances prétendues tardives..., Paris 1764. Die Kontroverse ist dargestellt in: Paul Delaunay, Le monde médical Parisien au dix-huitième siècle, Paris 1906, S. 431-440.

${ }^{53}$ Louis (Anm. 52), S. 88; Delaunay (Anm. 52), S. 435-439. Mit Louis waren einig der Venerologe und Gynäkologe Jean Astruc (1684-1766) und der Göttinger Medizinprofessor Rudolf Augustin Vogel (1724-1774) mit seinem Doktoranden Johann Christoph Harrer (Dissertatio inauguralis medica de partu serotino valde dubio, Goettingae 1767).

${ }_{54}^{5}$ [Jean Lebas (Le Bas),] Question importante. Peut-on déterminer un terme préfix pour l'accouchement?, [Paris] 1764. 
${ }^{55}$ Delaunay (Anm. 52), S. 431-440; weitere Gegner Louis': Achille-Guillaume Le Bègue de Presle, Jean-Baptiste-Louis Chomel († 1765) und Jacques-René Tenon (1724-1816), der berühmte Chirurg.

${ }^{56}$ A[ntoine] Petit, Recueil de pièces Relatives à la question des Naissances tardives..., Amsterdam 1766.

${ }^{57}$ Lebas (Anm. 54), S. 110, 112.

${ }^{58}$ Albrecht von Haller, Vorlesungen über die gerichtliche Arzneiwissenschaft. Aus einer nachgelassenen lateinischen Handschrift übersezt, 2 Bde., Bern 1782, 1784. [Vorlesung, gehalten in Göttingen im Sommersemester 1751, Nachschrift von Gottlieb Emanuel von Haller]; Bd. 1, S. 108, 119. Damit übereinstimmend: Haller, Primae lineae physiologiae... [Editio secunda], Gottingae 1751, S. 557.

59 Albrecht von Haller, Elementa physiologiae corporis humani, Bd. 8, Bernae 1766, S. 421429. Eine ähnliche Auffassung vertrat Haller bereits 1744 im Kommentar zu Boerhaaves Vorlesungen (Haller [Ed.], Hermanni Boerhaave Praelectiones academicae ..., Tom. V. Pars II., Gottingae 1744, S. 490-495, besonders S. 494) und auch wieder in späteren Jahren (Haller, Erster Umriß Der Geschäfte des Körperlichen Lebens ..., Berlin 1770, S. 510).

${ }^{60}$ Louis (Anm. 52), S. 32-36.

${ }^{61} \mathrm{~F}$ [rançois] E[mmanuel] Fodéré, Traité de médecine légale et d'hygiène publique, ou de police de santé, Bd. 2, Paris 1813, S. 108-140, 180-204; Fodéré, Naissances précoces et naissances tardives, in: Dictionnaire des sciences médicales, Bd. 35, Paris 1819, S. 152-173.

${ }^{62}$ Friedrich Benjamin Osiander, Handbuch der Entbindungskunst, Ersten Bandes erste Abtheilung, Tübingen 1818, S. 337-359.

${ }^{63}$ Arnold Adolph Berthold, Über das Gesetz der Schwangerschaftsdauer, in: Abhandlungen der Königlichen Gesellschaft der Wissenschaften zu Göttingen, 2. Bd. (1842-1844), Göttingen 1845, S. 181-224; Zitat S. 184.

${ }^{64}$ Paul Loewenhardt, Die Berechnung und die Dauer der Schwangerschaft, in: Archiv für Gynaekologie, 3. Bd., Berlin 1872, S. 456-491; Zitat S. 476.

${ }^{65}$ Max Hasler, Ueber die Dauer der Schwangerschafi, Diss. med., Zürich 1876, S. 43.

${ }^{66}$ Heinrich Fasbender, Geschichte der Geburtshülfe, Jena 1906, S. 495f. Wegen der Unsicherheit des Konzeptionstermins mußte meist von der Menstruation ausgegangen werden. Dabei war 1906 noch ungewiß, ob das Ei der letzten eingetretenen Periode oder das der ersten ausgebliebenen befruchtet werde.

${ }^{67}$ Franz Karl Nägele, Lehrbuch der Geburtshülfe für Hebammen, Zweite, vermehrte und verbesserte Auflage, Heidelberg 1833, S. 81; in der ersten Auflage, Heidelberg 1830, fehlt die Regel.

${ }^{68}$ Pschyrembel (Anm. 23), S. 17.

${ }^{69}$ Hermann Knaus, Die Physiologie der Zeugung des Menschen, 3.Auflage, Wien 1950, S. 159-170; S. 404-420: «Zur Geschichte der Erforschung des Konzeptionstermines.»

${ }^{70}$ Hermann Knaus, Die wahre Dauer der menschlichen Schwangerschaft. Zum Ende der Berechnung des Geburtstermins nach F.C.Nägele, Wien 1970, 160 S.

${ }^{71}$ Carl Gustav Carus, Lehrbuch der Gynäkologie ..., Zweiter Theil, Leipzig 1820, S. 87-89; 3.Aufl., Zweiter Theil, Leipzig/Wien 1838, S. 79f. - Eine ähnliche Tabelle findet sich in Dietrich Wilhelm Heinrich Busch und A. Moser, Handbuch der Geburtskunde ..., 1. Bd., Berlin 1840, S. 361-369. 
72 [Adam Elias von Siebold,] Kluge's Schwangerschafts-Kalender ..., in: Journal für Geburtshülfe, Frauenzimmer- und Kinderkrankheiten, 6.Band, 1826, S. 127-135 und Tafel am Schluß des Heftes. Erwähnung bei Fasbender (Anm. 66), S. 252; Kluge wird dort fälschlicherweise als Erfinder des Schwangerschaftskalenders überhaupt bezeichnet.

${ }^{73}$ Medizinhistorische Sammlung der Universität Zürich, Inventarnummer 1052. Lackdose mit aufgesetztem Deckel, Höhe 2,5 cm, Außendurchmesser 10,7 cm; kein Herstellervermerk. - Dosen dieser Art konnten «bei Herrn Feder in Berlin, Mittelstraße No. 12 par terre» für 1 Thaler 16 Groschen oder bei Stobwasser in Berlin für 2 Thaler preuß. Courant bezogen werden (Journal [Anm. 72], S. 135, 478). Vgl. dazu Clare Le Corbeiller, Alte Tabakdosen aus Europa und Amerika, München (1966), S. 109-115: Stobwassersche Lackdosen aus Papiermaché sehen der unserigen ähnlich, sind jedoch gewöhnlich innen auf dem Deckel signiert.

${ }^{74}$ W[illiam] Tyler Smith, A Manual of Obstetrics ..., London 1858, S. 229; eine genaue Beschreibung fehlt im Text.

75 Bernhard Sigmund Schultze, Lehrbuch der Hebammenkunst, 3. Aufl., Leipzig 1870, S. 74f., Fig. 35.

${ }^{76}$ Alexandre Lacassagne, Schéma du développement du produit de la conception et calendrier de la grossesse, Paris 1886. Abgebildet in: J. Voituriez, Les calendriers de la grossesse, in: Archives de Tocologie..., Bd. XVII, 1890, S. 285-293.

77 Pierre-Victor-Alfred Auvard, Le Nouveau-né, Paris 1890 (nach Voituriez [Anm. 76], S. 291, Abb.).

${ }^{78}$ Nach Voituriez (Anm. 76), S. 290, Abb.

${ }^{79}$ The American Journal of Obstetrics ..., Bd. XXII, 1889 [Beilage zum Novemberheft, ohne Kommentar].

${ }^{80}$ In Klammern ein offenbar beim Abschreiben versehentlich wiederholter Passus.

${ }^{81}$ Wie auf S. 1 dargelegt ist, sind je sieben Tage mit a bis g bezeichnet, was das Abzählen der Wochen erleichtert.

${ }^{82}$ Stychlin, kleiner Stiel; in Muralt (Anm. 24), S. 62, heißt es «Stielein».

83 Über die Ernährung des Fötus durch das Fruchtwasser siehe: Joseph Needham, $A$ History of Embryology, Second Edition, Cambridge 1959, S. 180.

${ }^{84}$ Auch die Unterschrift ist kopiert.

\section{Summary}

The author describes a pregnancy calendar mounted on a painted panel, which the Zurich town physicien Johannes von Muralt (1645-1733) presented, in 1701, to the «Ehegericht», a court for moral offences, marital cases, etc. This calendar is now in the Museum of the History of Medicine in the University of Zurich. It is based on the assumption of a gestational period fixed to 278 days. Von Muralt's directions for use are transcribed and analysed. Views on the normal duration of gestation remained contradictory down to the 19 th century; they are rewieved briefly. Finally some other pregnancy calendars are discussed.

Dr.med.Urs Boschung

Konservator

Medizinhistorisches Institut

Rämistraße 71, 8006 Zürich 